\title{
Tumelo le Moruo
}

Author:
Vuyani S. Vellem ${ }^{1}$
Affiliation:
'Department of Dogmatics
and Christian Ethics,
Centre for Public Theology,
University of Pretoria,
South Africa
Correspondence to:
Vuyani Vellem
Email:
vuyani.vellem @up.ac.za
Postal address:
Private Bag X 20, Hatfiled
0028, South Africa
Dates:
Received: 30 July 2014
Accepted: 04 May 2015
Published: 08 Oct. 2015
Copyright:
Co 2015. The Authors.
Licensee: AOSIS
OpenJournals. This work is
Vellem, V.S., 2015 , 'Tumelo le
Moruo', Verbum et Ecclesia
36(1), Art. \#1373, 6 pages.
http://dx.doi.org/10.4102/
ve.v36i1.1373

Author:

Vuyani S. Vellem

\section{Affiliation:} and Christian Ethics, Centre for Public Theology, University of Pretoria, South Africa

Read online:

Paleng ya rona batho ba batsho, tumelo ya boKreste e fihlile lefatsheng la rona la Afrika Borwa mmoho le dikgoka tsa ditjhaba tsa boPhirima. BoKreste bo fihlile ka nako ya dintwa tseo mohopolo wa tsona e neng e le ho hapa lefatshe la, batho ba batsho. Ka mantswe amang, rona batho ba batsho, re ile ra qetella re le setjhaba se ileng sa hlolwa, mme lefatshe la rona la nkuwa ka dikgoka. Ka hare ho dikgoka tsena, ho ne ho dutse tumelo ya boKreste. Makgowa a ile are: 'A re kwaleng mahlo re rapeleng, rona ra kwala mahlo, mme ha re qeta hore Amen, re bula mahlo, ra fumana lefatshe le nkuwe matsohong a rona ho setse Bibele.' Re ile ra sala le Bibele eo ka yona re lekileng dilemong tse fitileng ho lwana ntwa ya topollo, kapa tokoloho hofihlela selemong sa 1994. Le ha re ile ra fumana tokoloho ka selemo seo, hare so ka re lokoloha ho tsa moruo. E kaba sena se bolela eng mabapi le tumelo ya rona ya boKreste? Segolweng sena re leka ho araba potso ena. Tumelo ke eng ho batho ba sa lokolohang moruong wa naha ya bona? Re lekola pale ya boKreste, tumelo ya batho le maemo a kereke ntlheng ya ho tadimana le tokoloho ka tumelo.

Faith and economics. In our history from a black perspective, Christianity arrived through violent conquest from the west. Evidentially, this faith coincided with wars of dispossession and the ultimate defeat of black Africans. It is difficult to separate the violent defeat of black Africans from the arrival of Christian faith. This well-known statement within the circles of black Theology of liberation: When the white man arrived in our land he said, 'let us pray and after prayer, when we opened our eyes, our land was taken and only the Bible was left in our hands,' captures the black sentiment of this history. Ironically, it was this Bible that black Africans used to wage their struggle for liberation up to the demise of apartheid in 1994. Nonetheless, political liberation is not enough as the struggle for economic liberation continue in South Africa post 1994. In the light of this history, what then is the role of faith for a people politically liberated without economic liberation? This article examines this history from the perspective of black faith and its role for liberation.

\section{Mantswe a Bohlokwa: Dikgoka, kereke, tumelo, tokoloho}

\section{Selelekela}

Taba ya rona haholoholo e maloka le tsela eo re arohanyang tumelo le moruo ka yona. Re leka ho hlalosa hore ha se nnete thutong ya boKreste ho arohanya moruo le tumelo. Ke hore, le dipolotiki, kapa dikarolong tsohle tsa bophelo, ha se nnete ho arola tumelo le mafapha, kapa dikarolo tsa bophelo ba batho. Re qala ka ho lata pale ya ho fihla ha boKreste lefatsheng la rona la Afrika Borwa. Sepheo sa rona hase ho pheta ho ngola pale ena empa, ho leka to totobatsa tsela eo tumelo ya boKreste, ho fihleng ha yona, e sa kang ya arohana le moruo ka teng. Paleng ena, ho hlakile hore ho ile haba le mefuta e fapaneng ya dikereke metsong le tshimolonhong ya boKreste ha di fihla lefatsheng la rona. Kamora pale ena, re sheba ka bokgutswanyane, tumelo ya batho ba batsho mme ho latele kereke ya batho ba batsho mohloding wa tumelo ya bona. Dikarolo tsena tsa pele ke mohlodi oo ka ona re tadimang dilemo tsa tokoloho Afrika Borwa mabapi le seo kereke ka bophara e lokelang ho tadimana le sona tumelong ya batho ka bophara. Tjhe, ha jwale, moruo le tumelo e sa le qaka. Matla a rona re le badumedi, ho hlanaka taba ena, a mofuteng wa 'kereke ya lefatshe kaofela' e hlotsweng metsong ya tumelo ya batho ba batsho kapa batho ba hatellestweng.

\section{Pale ya Batho ba Batsho le Tumelo ya Bokreste}

Hare tadima ho fihla ha batho ba basweu Afrika Borwa, mme re qala ka ho hata ha Jan Van Riebeeck motseng wa Kapa, re lokela ho hopola hore sepheo sa bona ene e le sefe. Haholoholo, seo ba neng ba se labalabela e ne e le ho aha kou ho thusa dikepe tse neng di fetela lefatsheng 
la India leo ba neng ba hweba le lona. Ka mantswe a mang, sepheo ene e le kgwebo. ${ }^{1}$ Ka mora hore lefatshe la Holland le dumelle kopo ya Jan Van Riebeeck ya ho aha kou ena Kapa, diketsahalo tse latelang jwalo ka ho lema le ho hlahisa veine, di ile tsa eba ditla-morao tsa sepheo sa ho hweba ha bona le India.

Monahano wa hore e be baipei lefatsheng lena le ona o ile wa latela, mme ho hlongwa ha dikereke le hona ka tsela e jwalo. Re leka mona ho bopa setshwantsho sa ho fihla ha tumelo ya rona ka pale ya ho fihla ha batho ba basweu Afrika Borwa. Ho fihla ha batho ba neng ba dumela ho Jesu, ke ditla-morao sepheong sa ho ntshetsa pele kgwebo ya Makgowa le lefatshe la India, pele monahano wa ho jala tumelo o fihla. Mothating wa ho ipea le ho atisa bodulo ka ho lema morara, ho ile ha bonahala hore jwale ho hlokahala hore ho eketswe lenane la baipei Kapa. Dikepe tse neng di ba tlisa di ile tsa latela, mme jwalo ka ha bantse ba eketseha, hwa hlokahala hore ba fumane tsela ya ho anetsa tumelo ya bona. Diphutheho tsa pele Afrika Borwa, di ne di thusa ho fepa baipei ba Makgowa le malapa a bona moyeng. ${ }^{2}$

Potso ya bohlokwa e lateleng ke hore jwale, balemi ke bo mang? Efela ba ahi ba Afrika Borwa, baKhoisan, ba ile ba etswa basebetsi badipolasi tsa morara, le tsa veine. Ha nako e ntse e tsamaya, ho ile ha bonahala hore ho hlokahala basebetsi babangata ho kotula morara le ho ritela veine. Ho ile ha fihla sekepe seneng se jere makgoba ho tswa mafatsheng a tshwanang le Malaysia, ha re etsa mohlala. Pale ena e ya tsejwa, sa bohlokwa ke hore re tlameha ho hopola hore kannete, bothata bo qadile mona. Baipei ba ile ba nka lefatshe ka dikgoka le dikgomo kapa moruo wa batho ba batshodintho tsohle tsa badudi- qetellong batho ba batsho ba ile ba sebediswa jwalo ka makgoba mofuteng o fapaneng wa kgwebo ya boPhirima.

Tumelo ya boKreste, e fihlile pele motseng wa Kapa ka sepheo sa ho fepa moya wa baipei naheng ya rona. Setshwantsho seo re ka se sebedisang mona ke hore kereke ya pele, kapa mofuta wa kereke eo re buang ka yona ha jwale, ke kereke ya baipei. Ha re tswela pele re kare, ke tumelo ya baipei. Ka seNyesemane re kare Settler Christain faith, or Settler Church. Ho phatlalatsa tumelo ya boKreste, e ile ya eba mosebetsi wa di-Missionary. Bothata ke hore di-Missionary ka ho phatlalatsa tumelo di fetotse tumelo tsa e koponya le sepheo sa kgwebo mme ka tsela ena, tumelo e hasitswe jwaloka haeka batho ba batsho e ne e le 'mobu' o nonneng, wa ho hasa tumelo e neng e tswakile sepheo sa moruo le ho hapa lefatshe. Hona ho bolela hore ho ile haba le mofuta o mong wa kereke- kereke ya ho phatlalatsa evangedi bathong ba batsho. Mofuta ona he ke wa dikereke tse hlotsweng ke diMissionary (Missionary Initiated Churches). Mofuta ona, o ile wa tswaka tumelo le kgwebo, haholo-holo e le ho sebedisa tumelo e ntjha lefatsehng la rona — boKreste-ho bebofatsa

1.Tadima dibuka tsena tse latelang ho ikgopotsa pale ena: De Gruchy (2005); Hinchliff (1968); Cochrane (1987); Le Saayman (2008).

2.Mohlala ke phutheho ya pele ya kereke e neng e bitswa Presbyterian Church in South Africa. Phutheo ya bona ya pele Kapa, e ileng ya thehwa ka selemo sa 1827 ene e ele ya batho bao e eneng e le masole aManyesemane. tsela ya ho fumana basebetsi mapolasing a baipei a veine le ho hapa lefatshe.

Ke rata hore pele re fetela pele, re ke re hlakise taba ya bohlokwa haholo. Kereke ya baipei, enee thusa baipei moyeng hore sepheo sa kgwebo ya bona se tswelle pele. Kereke ya diMissionary yona? Kopano mahareng a mefuta ena e mmedi ya dikereke ke eng? Mefuta ena: 'kereke ya baipei' le 'kereke ya di-Missionary,' mohlomong e utlwahala hantle hare re tumelo ya boipei le tumelo ya phatlalatso ya boKreste, ke eng? Tumelo ya boipei le tumelo ya di-Missionary? Ha eba hona le kopano mahareng a tumelo le moruo, mofuta wa kereke ya baipei, ka ho totobala, ke kereke e nang le maqhama le moruo. Re tlameha ho dumela hobane ena ke nnete ya pale ya rona ya ho fihla ha tumelo ya boKreste naheng ya rona. Ka tsela e tshwanang, re kare, ena ya di-Missionary, le yona e fihlile ya phatlalatsa tumelo ka setso, meetlo, mekgwa le puo tse fapaneng le setso, meetlo, mekgwa le puo ya batho ba batsho. Re lokela ho bea taba ena hantle.

Mothating wa hona jwale, taba ya bohlokwa ke phapano mefuteng ena ya dikereke tseo re ngolang ka tsona, pale ya tsona e tebileng, ke taba e fapaneng. Mohlomong ho a hlokahala hore re re, ho na le ditla-morao tse lokileng le tsa sa lokang paleng ya rona ka baka la mefuta ena e fapaneng ya dikereke. Hopola mohlodi wa rona motsotsong ona. Ha e le ha jwale, seo re buang ka sona ke hore, mefuta ena ya dikereke, e hlalosa maqhaama a tumelo le tsela eo kereke e hlotsweng ka yona Afrika Borwa. Ha re shebisisa tsela eo tumelo ya boKreste e hasitsweng, ya phatlalatswa ka yona, re fumana hore di-Missionary di ile tsa sebedisa boKreste ho hapa naha le ho hatella badudi ba naha ena ho fihlela nakong tsa mmuso wa Kgethollo (apartheid).

Taba ke ena: pele ho fihla mmuso wa Kgethollo, hone ho se hona le dikereke tseo tumelo ya tsona eneng e sena boikemisetso ba ho ntshetsa pele bophelo ba batho ba batsho. Dikereke tsa baipei, di ile tsa kgetholla batho batsho. Mohlodi wa tumelo ele kgwebo. Dikereke tsa di-Missionary tsona di ile tsa phatlalatsa tumelo ya boKreste ka ho ikopantsha le mebuso eo e neng e shebile ho hapa lefatshe. Ka mantswe amang di-Missionary, di ile tsa bebofatsa ntwa ena pakeng tsa batho ba batsho le dinaha tsa bophirima ka tumelo, sepheo sa tsona e le kgwebo le ho hapa lefatshe. Pele ho fumanwa taemane le gauda, ho ne ho se ho na le thuto ya boKreste eneng e bistwa 'Evangedi ya Mosebetsi', Gospel of work. Charles Villa-Vicencio o hlalosa thuto ena bukeng ya hae (1989). Thuto ena, e bitswang 'Evangedi ya Mosebetsi' e ne e le thuto ya boKreste, sepheo sa yona ele ho sebedisa boKreste, ho sokolla batho ba naha ya rona hore ba amohele bo Kreste mme ka tsela eo ebe basebeletsi ba ditjahaba tse neng di ikgapela lefatshe la rona. ${ }^{3}$ Mefuta ena e mebedi ya dikereke, e ne e sa natse ditlhoko tsa batho ba batsho. Tumelo e nang le maqhama a kgwebo, le tumelo e nang le maqhama a ho sokolla le ho bolaya setso, mekgwa le meetlo

3. Ha o bala buka ena, Duncan (2003) o fumana botebo ba pale ena. Mongodi wa yona o totobatsa mekgwa eo dimishinari di ileng tsa hatella dikellello tsa batho di yona o totobatsa mekgwa eo dimishinari di ileng tsa hatella dikellello tsa batho di
sebedisa tumelo ya boKriste. Sheba hape bangodi bana: Maluleke (1997); le Vellem (2013a). 
ya bophelo le dipuo tsa batho ba batsho, ke mefuta ya tumelo ya matlokotsi lefatsheng lena; qaka kannete matsatsing a ho phela ha rona. Mefuta ena ya dikereke, re ka e tshwantsha le lesaka. Lesakeng la tumelo ya baipei ke kgwebo, ha lesakeng la tumelo ya phatlalatso ya boKreste, e le ho ptjhatla tswelopele ya batho ba batsho.

Ho ile ha ba le mofuta wa boraro! Batho ba naha ena, ba ahi, ka ho bona hore ha bana tulo dikerekeng tsena tse pedi tseo re dihlalositseng ho fihla ha jwale, ba ile ba tswa ho qala mofuta wa kereke o atametseng ho ditlhoko tsa bona. Paleng ya dikereke tsa Afrika Borwa, ona ke mofuta o bitswang Topia, (the Ethiopian Movement), Dipostola kapa dikereke tsena tsa Sione. ${ }^{4}$ Ho tswa ha batho ba batsho dikerekeng tsena re kare ebile maqhubu a ileng a phatlalla naha ka bophara. Ho bonahala hore kgethollo ka mmala, ho shebela fatshe ha setso sa batho ba batsho, dintwa tse neng di hapa le fatshe, tumelo e kgethollang, ebile a mang a mabaka a ileng a etsa hore batho ba batsho ba amohele tumelo ya boKreste empa kantle ho mefuta ela e mebedi eo re buileng ka yona. Qaka mabapi le tumelo yaBokreste bathong ba batsho e qadile mona. Batho ba batsho ha baka ba qhala tumelo ena kaofela ba e kwalla kantle ho lesaka la bona. Ba ile ba e amohela le ha ba sa ka ba amohela thuto ya tumelo e hapang lefatshe la bona, le e hatellang kelello ya motho.

Pale ka tumelo ya rona le ho tla ha kereke lefatsheng lena e tebile. Maikutlo a Dirk Smit (2007:61-72) mabapi le hore kereke ke eng, a bohlokwa haholo. Lentswe lena 'kereke', le bolela dintho tse ngata matsatsing a ho phela ha rona. Ho batho ba bang kereke ke 'phutheho'. Phutheho haholoholo ke moo batho ba rapelang mmoho teng. Ka manstwe a mang, mofuta wa tshebeletso phuthehong ka nngwe, o hlalosa ka moo batho babang ba utlwisisang 'kereke'. Tsela e nngwe ke eo dikereke tse fapaneng jwalo ka kereke ya Wesele, kereke ya Roma, jwalo-jwalo di bitswang ka teng. Ho latele lefapha kapa mafapha a dikereke jwalo ka South African Council of Churches (SACC), mofuta oo re ka reng ke 'kereke ya kakaretso'. Smit o thusa ho hlalosa mefuta ena e meraro eo re buileng ka yona. Diphuthehong, dikerekeng tse tshwanang le Wesele, Roma, Lutere, jwalo-jwalo, mafapheng a fapaneng a dikereke, kerekeng ya kakaretso, mefuta ena e meraro eo re buileng kayona e bonahala mofuteng wa tumelo e rutwang le ho haswa ke mefuta e hlaloswang ke Dirk Smit. Ka mantswe a mang, ha re eso ka re lahleha ho ya ka moo re hlalositseng pale ya rona ho fihla ha jwale. Maqalong; kgwebo, kgatello ya setshaba e le ha ho hlokwa basebetsi bao ho neng ho le bonolo feela hoba hapa le hoba etsa makgoba, e bile mosebetsi of moholo wa kereke.

John de Gruchy (2014) le yena o a re thusa ha re sheba tsela eo a hlalosang mokgwa oo dikereke di ipatlang ka ona. Boitsibi ba dikereke kamora dilemo tsa kgatello ke qaka empa, le yena ke nahana hore a keke a hana tlhaloso ya rona ya mefuta ena e meraro ya kereke, haholoholo tumelo e tsamaisanang le mofuta ka mong ho tswa paleng ya ho fihla ha boKreste Afrika Borwa.

4.Pale ena e fumaneha dibukeng tse ngata tse ngotsweng. Sheba, Anderson (2000).
Yena o dihlalosa ka mantswe ana: ya baipei, ya bahapi, ya di-Missionary, ya merabe kaofela, le ya lefatshe kaofela (De Gruchy 2014:17). ${ }^{5}$ Tsela e phatlalletseng eo a hlalosang mefuta ya dikereke ka ho fapana ha tsona le yona e ya thusa. Jwalo ka ha re se re hlalositse, ha hona bothata tseleng tsena tseo kereke e hlaloswang ka tsona. Mohlodi wa taba ke hore ho tsona kaofela, metso ya mefuta ela e meraro, baipei, diMissionary le ya batho, le metso ya tumelo e hasitsweng lefatsheng la rona, e teng ho mefuta ena kaofela- mefuta ya Smit le De Gruchy, le tsela eo ba hlalosang lentswe lena: 'kereke'.

Ho bohlokwa hore re shebe taba ena ka bophara ba yona, hore botebo ba lenstwe lena, 'kereke' le tumelo e tsamaisanang le mofuta ka mong wa yona re e utlwisise hantle. Paleng ena ya ho tla ha boKreste Afrika Borwa, re etsa phapano mabapi le mefuta e meraro ya kereke, mme le botebo ba phapano mabapi le tumelo e tsamaisanang le mofuta ka mong. Lesaka la mofuta ka mong le kentse dikahare tsa tumelo le ho fapana ha yona le tseding tumelong ya boKreste ka bophara ba yona. Jwale, lesakeng la batho ba batsho re ka bona eng?

\section{Tumelo ya Batho ba Batsho}

Jwalo ka ha re bona ho fihlela jwale, batho ba batsho ba ile ba bona hore ba bope tsa bona dikereke. Ka bokgutshwanyane, kereke tse hlotsweng ke batho ba batsho di ne di nka karolo ka tsela tse fapaneng ho lwanela tokoloho le topollo ya batho ka tumelo. Ka selemo sa 1912, ha ho ne ho thehwa African National Congress (ANC) Mangaung, dilemong tseo, mokgatlo ona o ne o bitswa South African Native Convetion (SANC), baetapele ba bangata e ne e le baetellipele ba dikereke. ${ }^{6}$ Ke kamora hore mane Sharpeville, ho bolawe batho ka sehloho bongodi ba tumelo ya batho batsho bo ileng ba teba haholo. Re tshwanetse hape ho hopola hore dilemong tsa 1960-1968, dikereke tsena: Wesele, Congregational, Prebyterian, Tjhatjhe, di ile tsa qala sekolo sa ho kwetlisa baruti Alice, se neng se bitstwa Federal Theological Seminary (Fedsem). Pale ya sekolo sena ka botebo e bontsha hore tumelo le kgatello ya batho e ile ya eba sesosa sa ho thehwa ha sona (Denis \& Duncan 2011). Athe ka 1968, Lefapha la dikereke la Afrika Borwa (South African Council Churches) (SACC), le ile la thehwa e le ha ho ne ho fetolwa lefapha la diMissionary ka baka la diketsahalo tsa Sharpeville, maemo a batho ba batsho lefatsheng la bona, le bokgopo ba kgethollo. Ho ngolwa-bongodi ka botebo ba tumelo ya batho batsho bo hlahile hantle dilemong tsena. E ne e le dilemo tsa 'Black Consciousness', (Mohopolo/kelello ya botsho: e toboketsang ho itseba, ho ipatlisisa, eo e bileng karolo ya bongodi ba thuto ka tumelo ya batho ba batsho).

E mong wa bangodi ba tumelo ya batho ba batsho, Allan Boesak, ore tumelo ena ke ya maemo ao batho ba batsho ba phelang ho ona (Boesak 1977:17). Ke thuto ya tumelo e halolosang evangedi ya Kreste ka maemo a batho ba

5.Ka Senyesemane: Settler, Colonial, Missionary, Multiracial and Ecumenical, ka ho latellana ha tsona.

6.Peter Walshe (1995) o ngotse pale entle ka taba ena. 
hatelletsweng, batho ba batsho, batho ba senang letho. Tumelo ya batho ba batsho ha se tumelo e kgethollang empa ke tumelo e kgothatsang batho bao dikellelo, meya, le botho ba bona bo hatelletsweng ebile ho thwe ha bayo paleng ya botho lefatsheng kaofela. Ke thuto ya tumelo ya ho iphumana le ho ipatlisisa tumelong ya boKreste. Jwale ka ha re se re hlalositse, batho ba batsho ba ileng ba bolawa ka sehloho Sharpeville selemong sa 1960, le diketsahalo tsa ho tshwana le Cottelsoe, ho thehwa ha Fedsem le SACC, ho ile ha hlahisa bangodi ba hotshwana le bo Manas Buthelezi, Simon Gqhubule, Khoza Mgojo, Basil Moore, Bonganjalo Goba, Allan Boesak, Itumeleng Mosala, Takatso Mofokeng, Buti Tlhagale, Mokgethi Motlhabi, Tinyiko Maluleke le babang. Le bo mme ba ile ba qallela ho ngola; Roxanne Jordan, mme Mmadipoane Masenya, Ngwn'a Mphahlele le Puleng Lenka-Bula, le babang, ka morao ha dilemo tsa kgethollo. Banna bana le basadi bana, ba ile ba ngola, ba qala thaloso ya bodumedi boKresteng ka maemo a batho ba batsho. Ba seolla kgethollo, ba qhaqholla mafito ao tumelo ya boKreste a ileng a tlamellwa ke baipei le di-Missionary ka yona. Ntho e ba kopanyang kaofela ke hore, tumelo ya boKreste ekeke ya arohangwa le tokoloho ya batho. Efela dilemong tsena, le mafatsheng amang moo ho neng hona le kgatello, jwalo ka Amerika le Amerika e Borwa, ho ile ha hlaha bangodi ba tshwanang le James Cone wa America, Gustavo Gutierrez was Peru, ba ruta ntho e lenngwe; tumelo ekeke ya arohangwa le tokoloho ya batho! Tumelo ke kotsi ha e hatella merabe, e kgetholla, e bile e tarakanya dikello tsa batho. Tumelo e nyatsang seriti sa batho ke kotsi. Ke tsela yeo mofuta wa thuto ya tumelo ya boKreste oo re buang ka wona o hlalosang boKreste ka yona.

Metsong ena ya bongodi ba thuto ka tumelo ya tokoloho le maemo a batho ba batsho, James Cone ore kereke ya batho ba batsho e bitseditswe matleng le leratong la Modimo ho ba ntho e lenngwe le matsapa a tokoloho ya batho (Stone 2012:218). Kereke ya Kreste ha e na kgethollo ka mmala le maemo. Hantle, kereke, ke kereke ya batho ba hatelletsweng. Gustavo Gutierrez le yena ore, jwalo ka sakramente ya pholoso, kereke, ke kereke ha e na le maqhama a tokoloho ya bao ba hatelletwseng lefatsheng (Stone 2012:223). Motso wa tumelo ya batho ba batsho, ke mofuta wa boraro ho mefuta eo e ileng ya hlaha ha ho fihla boKreste naheng ya rona. Ke tumelo eo bongodi ba yona bo ileng ba hlaka dilemong tsa bo 1968. Ke tumelo eo mafatsheng amang, e ileng ya bitswa ka hore ke tumelo ya tokoloho. Ke tumelo e hlakisang le tokoloho ya basadi kgatellong ya bona ke dikgoka tsa banna. Mohlodi wa rona ke mofuta wa tumelo le mofuta wa kereke. Kereke ena ya batho ba batsho e na le thuto ka tumelo ya ho lokoloha ha batho kgatellong e tsamaiswang ka tumelo. Lesakeng la tumelo ya batho ba batsho, ke tokoloho ka tumelo.

\section{Tumelo le moruo Afrika Borwa ka mora selemo sa 1994}

Mefuteng ena ya dikereke eo re buileng ka yona ha jwale, mohlodi wa taba ke hore tokoloho ya batho batsho dipolotiking, ha se tokoloho e felletseng. Ka mantswe amang, mosebetsi le mohwanto wa tokoloho o sa tswela pele.
Ho dintho tse ngata tse ileng tsa fetoha ka mora selemo sa 1994, re sa lebale tse ntle haholo-holo ho fela ha kgatello ya batho ba batsho, empa tokoloho ya rona ho tsa moruo ha e soka e fumaneha. Le leng la mabaka ke hore kutlwano mabapi le tumelo le moruo e thata haholo. Metso ya kutlwano pakeng tsa tumelo le moruo kapa kgwebo re tlameha ho e lekola paleng ya ho fihla ha boKreste eo re lekileng ho ngola ka yona ho fihlela mona. Sena se etsa hore re botse potso e reng; ekaba kereke ka mora dilemo tse ngata tsa kgatello, e sebetsa jwang kamora tokoloho ya setjhaba ho lwanela tumelo tseo masakeng a tsona ho nang le mekgwa ya kgatello le bokgoba? Re tla lekola ka bophara le ka potlako mefuta ya melao ya moruo hofihlela ha jwale Afrika Borwa.

Molao ona o neng o bitswa Growth, Employment, and Redistribution (GEAR), o ile wa makatsa setjhaba kaha o ile wa phatlalatswa ka mora hore mokgatlo o busang, ANC o ithekise ka tokomane e neng e bitswa Reconstruction and Development (RDP) ka nako ya dikgetho tsa pele ka morao ho dipuisano tse tlisitseng apartheid mafelong. RDP e hlaloswa jwalo ka tokomane e neng e hatella tokoloho ya batho ho aba moruo ka toka le ho aha setjhaba se neng se hatelletswe ka ntle le qea-qeo. Tokomane ya RDP tjhebelo-pele ya yona ke ho lokisa tshenyeho e qadileng ha ho fihla batho babasweu bao dilemong tse ngata ba ileng ba phela bophelo bo monate ha batho ba batsho bona ba sokola ka ditlhoko tse tshwanang le matlo, mosebetsi, thuto, mafapha a bophelo le tseding. Kgethollo (apartheid), e ile ya etsa ka ho phethahetseng hore tsebo, moruo le matla di be matsohong a batho ba basweu feela. RDP maikemisetso a yona, e bile ho tobana hantle le ditholwana tsa kgatello ya batho mme ka bokgutshwanyane, setjhaba se ile sa e labalabela haholo.

Ka bomadimabe, puso ya ANC e soka e ya le hole, ho ile ha phatlalatswa molao wa GEAR. Mokgwa oo GEAR e ileng ya phatlalatswa ka wona le jwale o sihile batho ba ahlame! Mekgatlo ya ho hwanta ya basebetsi e lekile ho botsa dipotso e hlanaka taba ena, le kajeno taba ya moruo le melao ya oona e sa le qaka. Monghadi Nelson Mandela, o ile a jwetsa mekgatlo ena, le setjhaba hore GEAR ke molao wa mmuso wa ANC. Phapang mabapi le RDP le GEAR ke eng? GEAR ke molao wa moruo o tshepisang batho ditholwana tsa moruo ka ho hodisa moruo pele. Ka mantswe amang, setjhaba se lokela ho ema tsi, ho hodiswa moruo pele se ntse se le matlokotsing ha eba pale eo re qadileng ka yona re e latella hantle ho fihla mona. Sa bobedi, GEAR ke molao o ruisang barui hobane ka nako eo setjhaba se emetseng ho hola ha moruo, barui bona ba hodisa leruo la bona. Mabapi le GEAR, re ka fella mona, hobane mmuso o ile wa fetola molao ona jwalo ka ha ho buuwa ka New Growth Path (NGP).

NGP metsong ya yona, ha e na phapang haholo le GEAR. Vellem (2013b) o hlalosa tsela e tsamailweng ke mmuso ka melao ya moruo mme taba ya bohlokwa ke maikutlo a hae a reng haho ntho e ntjha e tlisistsweng ke NGP. Ka mora ho tloswa ha Monghadi Thabo Mbeki Polokwane, ha ho bonahale diphetoho tse tebileng mabapi le melao haholoholo ya moruo naheng ya rona. Monghadi Zuma o ile a ba a etela mafathse a hole jwalo ka Engelane le Amerika e le ha 
a tshepisa lefatshe hore phetoho ho melao ya moruo e keke ya ba teng. Manstwe ana ho tswa ho Vellem (2013b) a hlalosa sena tjena:

I read this policy framework not as a departure from GEAR, even though it is only the Accelerated and Shared Growth Initiative for South Africa (AsgiSA) that is mentioned in the quotation above. The pseudo-religious pedestal of neoliberal principles vividly remains. Faith in its crudest sense, in neoliberal economics must be repudiated. (p. 119)

Sena se hlalosa hore ha ho phapang mahareng a GEAR le NGP. Pele ho fihlwa ho NGP, ho kile ha ba le puo ka molao o mong o neng o bitswa Accelerated and Shared Initiative for South Africa (AsgiSA), oo le oona o neng o itshwanela le GEAR metsong ya ona. Mongodi ha se moithuti wa tsa moruo, empa taba ke ena: metsong ya GEAR, AsgiSA le NGP ke tumelo ea bohata ho moruo o hodiswang pele ha batho bona ba tlameile ho ema le ha e ba maemo a bona hase a thabisang. Ke moo mohlodi wa taba ya rona o tlamehileng ho dula teng. Tumelo ea bohata ho tsela eo moruo o laolwang ka yona! Ho fihlela dilemong tse mashome a mabedi, re ka re melao ya moruo, e hodisang moruo, ha e soka e thusa batho ba neng be hatelletswe kantle le batho ba mmalwa ba ileng ba ba le maqhama a puso ho fumana menyetla ya moruo. E mong wa bangodi ba tsa moruo Moeletsi Mbeki, o re puso ya ANC re tlameha ho utliwisisa ka ho hopola ka nako tsohle hore mokgatlo ona o busang, ha o na letho kantle ho matla a puso. Moruo, o matsohong a batho ba basweu.

Mohlomong ho bohlokwa hore re se fete mefuteng ena ya melao ya moruo pele re hlahloba ka bokgutshwanyane National Development Plan (NDP), eo ho buuwang ka yona matsatsing ana. Tokomane ya NDP, e tswela pele ka merero ya melao ena eo re seng re buile ka yona. E hlalosa hore naha ya rona e tlamehile ho ba lehae la rona kaofela, mme bofutsana bona, e be ntho ya maoba le maobane. Tokomaneng ya pele e bitswang Diagnostic Report, e ileng ya phatlalatswa ke ba komishinara ba NDP, e supa mathata a tshwanang le mosebetsi, thuto ya batho ba batsho, maemo a moruo le tse ding. Jwalo ka ditokomane tse ding tse tobaneng le moruo, le yona NDP, e tobile nthong e le ngwe: ekaba ho hola ha moruo qetellong o thusa jwang ho tlisa toka lefatsheng leo ba bang ba ileng ba hatellwa mme le tumelo ya boKreste e sebedisetswa kgatello ya bona ha ba bang bona ba ja monono? Ka nnete NDP le yona, metsong ya yona, haholoholo ebile ha re lebella maikutlo a fapaneng ka tokomane ena, (Vellem 2014:8-9) e tshepisa batho ba sokolang dintho tse kekeng tsa fumaneha mohlomong e bile le ka mora dilemo tse setseng pele ho selemo sa 2030. Kannete, le yona NDP e tebisa maikutlo le tumelo ya batho ho mofuta wa moruo o tlamehang ho hola ha barui bona ba tswela pele ka maphelo a bona empa bafutsana ba dutse ba kganngwe le ke ho bolawa ke tlala.

Ho fihlela ha jwale re lebelletse melao le ditokomane tsa, GEAR, NGP le NDP. Seo re se hlahlobileng haholo ke metso ya melao ena. Metso ya tsona e ya tshwana hobane e tshepisa tokoloho ka ho hodiswa ha moruo wa naha. Dilemong tse mashome a mabedi jwale, se bonahalang ka ho totobala ke hore le ha moruo o hola, batho ba fumanehileng ha ba kotule letho. Se etsahalang ke hore maemo a batho ba fumanehileng a mabe ho feta dilemong tse fetileng ha barui bona ba dutse ba kotula moruong wa lefatshe leo ba le hapileng ka dikgoka. Taba ena hase ya Afrika Borwa feela. Ha re lebella tumelo ya Accra, ya World Communion of Reformed Churches (WCR), re sheba le tokomane ya World Council of Churches, AGAPE, mofuta ona wa moruo, ke taba ya tumelo jwale. Batho lefatsheng ba kgodiswa hore moruo ka sekgowa, capitalism, o hodiswang pele ho ka abelwana ka ona, ke 'evangeli' ya Jesu. Re tlameha ho hopola hore hodiuwa ha lerako la Berlin, ho wa ha Russia le mmuso wa apartheid, ke diketsahalo tse tobaneng le tsela ena ya moruo wa bokapitali. Emong wa bangodi ba tsa moruo, Loretta Napoleoni (2008), ore mothating wa ho fetoha ha dintho lefatsheng ha howa mebuso ya Russia le Afrika Borwa, le lerako la Berlin, bohlaswa ba tsa moruo bo ile ba totobala. O re tsela ya moruo wa kgodiso pele, mokgwa wa yona ke bohlaha ha re latela buka ya hae haholo-holo sehloho sa yona Rogue Economics.

Kereke dilemong tsena he e kae? Tumelong e fapaneng le mefuteng ya dikereke, ha ho so ka ho eba le mohwanto o matla hobane thuto ya boKreste e tswang bophirima e re ruta hore tsa semmuso le tsa moruo, di arohangwe. Kereke e tlameha ho sheba metso ya tumelo ya batho ba batsho.

Ho bohlokwa ho hopola hore ka bophara, dilemo tseo re fumaneng topollo ka tsona, ke nako eo moruo kapa kgwebo lefatsheng e etellestweng pele ke dimmaraka. Ketellopele ya dimmaraka e hatella le ho bolaya bophelo, hobane e laola setso, tumelo, tsebo, dipolotiki mme hodimo ha tsena, ebe e thuswe ka sesole le dikgoka ke mafatshe a nang le matla. Hopola hore phetoho ya lefatshe kamora howa ha lebota la Berlin, le ho fela ha matla ha Russia, e entse hore ho be le thuto ereng kgwebo lefatsheng etla etswa ka tsela e lenngwe feela. Ke ka hoo dimmaraka di fumaneng matla a tshabehang dilemong tsena moo ebileng le mebuso e bonahalang e sena matla ho laola matlo a hwebang a phatlalletseng ka lefatshe kaofela. ${ }^{7}$ Thuto e tlatsitsweng lefatsheng ke hore 'ha ho na tsela enngwe' ya kgwebo mme dinaha di qobellwa ho latela melao e etswang ke matlo a kgwebo le ho feta dinaha tse kopaneng (Lapavitsas 2014:10-13). Lefatshe le fetohile. Matla a tjheleteng, dimmaraka di hatelletse dikarolo tsohle tsa bophelo.

Ha re shebisisa kereke Afrika Borwa mothating ona, puo ya kereke; tsela eo dipolotiki di latelwang ka yona, mme ka tsela eo, melao ya moruo jwalo ka GEAR le NDP kaofela di bontsha hore qaka e kgolo e teng lefatsheng la rona (Vellem 2013b:113). Haholo-holo qaka ke tumelo. Kgwebo e jarwa ka tumelo mme ho latela tsela eo re shebileng mefuta ya dikereke, mohlomong ho nepahetse hore mofuta wa kereke eo tumelo ya yona e leng moruong e na le matla a maholo dilemong tsa rona tsa tokoloho.

7.Mona re leka ho bebofatsa taba e fumaneng dibukeng le mangolong a mangata jwalo ka Accra Confession le AGAPE ya WCC. Ho se ho na le dibuka tse ngotsweng mme ena, ke engwe ya tsona: Terreblanche (2012). 
Babang ba bangodi ebile, ba tshwanang le Allan Boesak, ba ana hore tsela ena ya dipolotiki Afrika Borwa, kabo yona ena le tumelo e sa lokang (Vellem 2013b:125). Moruo le tumelo, kapa kgwebo le tumelo dilemong tsena tseo re phelang ho tsona, ke qaka, ebile ke kodi-ya-ma-lla. Thutong ya tumelo ya batho, re tlamehile ho ema ka maoto hore re shebane le ntwa ena, ka tumelo.

\section{Pheletso}

Batho ba batsho re lokolohile ho busa naha ya rona. Re qakehile ho fumana tokoloho moruong wa naha. Pale ya ho fihla ha boKreste kajeno e phela tumelong ya rona. Lesaka la tumelo ha le so thubehe. Pale ena, e jerwe ke tumelo eo motso wa yona eleng kgwebo le kgatello. Ho utulla maqhama a tumelo le moruo, tumelo e metsong ya kereke ya batho ba batsho, ke tshepo e kgolo ka Kreste morena wa rona. A re jaleng metso ya tumelo ya rona ya boKreste botebong ba lesaka la tumelo ya tokoloho. Jwalo ka ha Evangedi ya Johanne 10:10 e re ruta, bophelo bo felletseng, ke thato eo Modimo, ka Kreste a e fang badumedi.

\section{Summary}

The main purpose of this article is to demonstrate that there is a relationship between faith and economics. The article does this by locating this relationship within the context of the conquest, colonisation and Christianisation of black African people. Proceeding from the famous statement: 'When the missionaries came, we African people had the land and they had the Bible. They said, "Let us pray," and when we opened our eyes after the prayer, they had the land and we had the Bible,' the article immerses itself within the tradition of black Theology of liberation. The relationship between faith and economics eludes many Christians, so by presenting a historiographical connection between faith and economics in South Africa, the point could be demontrated. There are three distinct models of Christianity and consequently three ecclesiological models in South Africa. These are the Settler, Missionary and African models of Christianity with their corresponding models of ecclesiology. The relationship of the Settler and Missionary models of Christianity and ecclesiology with economics is one major point that is argued. The African response to this reality, manifest in the Ethiopian Movement and the African Independent Churches, which later found expression in the development of black Theology of liberation, provides a picture of faith that in its very beginnings could not overlook the question of economics and thus economic liberation. In South Africa post 1994, in the development of economic policies after the demise of apartheid, the error of the church and in particular black Theology of liberation is to imagine liberation without economic liberation. Following this logic, the propulsion of economic policies that have shaped the public landscape in South Africa post 1994, cannot be viewed as unrelated to the faith assumptions related to the models of Christianity in this land. IEconomics does harness faith ostensibly in a positive or negative way. The example of Accra and Agape are offered to make this point. Overall, socio-political praxis is faith as shown in the history of Christianity in South Africa, and ipso facto, faith is a central question in economics.

\section{Tlaleho}

\section{Mesebetsi e amanang Leona}

Baqapi ba phatlalatsa hore ha ba na a lichelete kapa a botho kamano eo ka 'na eaba tsela e fosahetseng susumetsoa ba ka ho ngola sehloohong sena.

\section{Ditshupiso}

Anderson, A., 2000, Zion and Pentecost: The spirituality and experience of Pentecostal and Zionist/ Apostolic Churches in South Africa, Unisa Press, Pretoria.

Boesak, A., 1977, Farewell to Innocence, A Social-Ethical Study of Black Theology and Black Power, Raven, Johannesburg.

Cochrane, JR., 1987, Servants of power: The role of the English speaking churches in South Africa, 1903-1930, Raven, Johannesburg.

De Gruchy, J.W., 2014, 'The quest for identity in the so-called "mainline" churches in South Africa', in E.M. Conradie \& J. Klaasen (eds.), The quest for identity: In so-called mainline churches in South Africa, EFSA Series, pp. 15-31, Sun Press, Stellenbosch.

De Gruchy, J., 2005, The church struggle in South Africa, Minneapolis, Fortress.

Denis, P. \& Duncan, G., 2011, The Native School that Caused all Trouble: A Hisotry of the Federal Theological Seminary of Southern Africa, Cluster Publications, Pitermarizburg.

Duncan, GA., 2003, Lovedale: Coercive agency, Cluster, Pietermaritzburg.

Hinchliff, P., 1968, The Church in South Africa, SPCK, London.

Lapavitsas, C., 2014, State and finance in financialised capitalism, Centre for Labour and Social Studies, London.

Maluleke, T., 1997, 'Half a century of African theologies', Journal of Theology for Southern Africa 99, 4-23.

Napoleoni, L., 2008, Rogue economics: Capitalism's new reakity, Seven Stories Press, New York.

Saayman, W., 2008, Being missionary, being human: An overview of Dutch reformed mission, Cluster Publications, Pietermaritzburg.

Smit, D., 2007, 'On the impact of the church in South Africa after the collapse of the apartheid regime', in Conradie, E. (ed.), Essays in public theology, pp. 57-74, Sun Media, Stellenbosch

Stone, BP., 2012, A reader in ecclesiology, Ashgate Publishing Limited, Surrey.

Terreblannchce, S., 2012, Lost in transformation, KMM, Sandton.

Vellem, V., 2013a, 'The native experiment: The formation of the Bantu Presbyterian church and the defects of faith transplanted on the African soil', Missionalia 41(2), 146-162. http://dx.doi.org/10.7832/41-2-11

Vellem, V., 2013b, 'A critical black analysis of the Church's role in post-Apartheid struggle for socio-economic justice', Studia Historiae Ecclesiaticae December XXXIX(2), 113-130

Vellem, V., 2014, 'Modern slavery: A critical reading of the NDP and its promise for employment', Koers-Bulletin for Christian Scholarship 79(2), Art. \#2163, 8 pages. http://dx.doi.org/10.4102/koers.v79i2.2163

Villa-Vicencio, C., 1989, Trapped in Apartheid, David Philip, Cape Town.

Walshe, P., 1995, Prophetic Christianity and the liberation movement in South Africa. Cluster, Pietermaritzburg. 